\title{
siRNA-mediated MDM2 inhibition sensitizes human lung cancer A549 cells to radiation
}

\author{
WANFENG GUO ${ }^{1,3}$, KAZI MOKIM AHMED ${ }^{4}$, YANPIN HUI ${ }^{2}$, GUOZHENG GUO ${ }^{1}$ and JIAN JIAN LI $I^{4,5}$ \\ Departments of ${ }^{1}$ Radiation Medicine, ${ }^{2}$ Pathology, Fourth Military Medical University, Xi'an 710032; \\ ${ }^{3}$ Department of Oncology, 307th Hospital, Beijing 100071, P.R. China; ${ }^{4}$ Division of Molecular Radiobiology, \\ Purdue University School of Health Sciences; ${ }^{5}$ Purdue Cancer Research Center, \\ Purdue University, West Lafayette, IN 47907, USA
}

Received December 15, 2006; Accepted February 14, 2007

\begin{abstract}
MDM2 (murine double minute 2) is well-documented to play a key role in radiation response and tumor radiosensitivity, thus offering an attractive clinic drug target to enhance tumor sensitivity to anti-cancer radiotherapy. In this study, we designed and tested two siRNA fragments against human MDM2 in non-small cell human lung cancer A549 cells. Transfection of mammalian expression vector pUR/U6 containing either MDM2 siRNA1 or siRNA2 fragment was shown to reduce MDM2 mRNA levels by $72 \%$ and $31 \%$, respectively. Western blotting detected a similar inhibition of MDM2 protein levels in cells transfected with MDM2 siRNA1. A549 cells transfected with the expression vector for siRNA1 significantly decreased cell proliferation and rendered cells more sensitive to radiation. The basal apoptotic and necrotic cells, $1 \%$ and $2 \%$, respectively, detected among A549 cells were increased to $2.6 \%$ and $14.4 \%$ after $\gamma$-irradiation with 5 Gy. Transfection of MDM2 siRNA1 induced $30.1 \%$ apoptosis and $12.7 \%$ necrosis while combined treatment of siRNA1 and 5-Gy radiation increased apoptosis and necrosis to $45.9 \%$ and $15.2 \%$, respectively. These data provide the first evidence that specific siRNA fragment (MDM2 siRNA1) targeting human MDM2 mRNA is able to enhance lung cancer radiosensitivity.
\end{abstract}

\section{Introduction}

Lung cancer is the leading cause of cancer-related deaths in the world, accounting for 1.2 million new cases annually. Non-small cell lung cancer (NSCLC) accounts for nearly $80 \%$ of all bronchogenic neoplasms and is characterized by a particularly poor prognosis with approximately $90 \%$ of patients

Correspondence to: Dr Jian Jian Li, Division of Molecular Radiobiology, Purdue University School of Health Sciences, West Lafayette, IN 47907, USA

E-mail: jjli@purdue.edu

Key words: siRNA, MDM2, A549 cells, ionizing radiation, radiosensitivity dying within 5 years of diagnosis. Most NSCLC patients are inoperable at diagnosis and are treated with radiotherapy and/or chemotherapy. Currently, although radiotherapy is an important modality of lung cancer treatments, this therapeutic strategy alone could not improve efficiently the long-term survival rate. Therefore, to establish an effective treatment, novel drug compounds combined with radiotherapy are urgently needed. Specific molecular targets for enhancing radiation-mediated cell killing are heavily weighted toward the improvement of the cure rate for cancer (1-3).

It is now well established that, in addition to inducing nuclear DNA damage, therapeutic ionizing radiation (IR) can activate specific signaling transduction that can influence the overall cell survival $(4,5)$. Accumulated evidence suggest a strong correlation between IR-induced gene expression and cellular radiosensitivity (4-7). Radiation-resistant breast cancer $\mathrm{MCF}+\mathrm{FIR}$ cells that are derived from a long-term therapeutic regimen of radiation show elevated MDM2 expression (8). Using lung cancer cell lines with different radiosensitivities, we identified several potential candidate genes resistant to IR (9). Of particular interest among the up-regulated genes we have identified was MDM2 (murine double minute 2), a negative regulator of the p53 tumor suppressor, which was shown to be a critical component in response to IR (10). MDM2 expression levels were found to be increased in radioresistant non-small cell lung carcinoma A549 cells, indicating that MDM2 could be a suitable target for re-sensitization of radiation-resistant lung cancer cells.

MDM2 is an oncogene (11), and it promotes the growth of tumor cells (12). Amplification (13) or overexpression (14) of MDM2 has been found in a variety of human carcinomas, including NSCLC patients (15) with poor prognosis (16). Most importantly, MDM2 expression is found to affect cell response to radiotherapy and chemotherapy $(10,17)$. Therefore, MDM2 may serve as a potential target to enhance the tumor radiosensitivity.

siRNA has been extensively studied as a means of attenuating the expression of specific gene transcripts both in vitro and in vivo $(18,19)$. siRNAs are short, double-stranded RNA molecules that can target mRNAs with complementary sequence for degradation via a process termed RNAi (RNA interference). In this study, we applied vector-mediated delivery 
of two siRNAs to knock-down the expression of MDM2 gene in radioresistant human lung cancer A549 cells to investigate whether inactivating MDM2 can enhance the sensitivity to therapeutic dose of IR (5-Gy $\gamma$-ray). Our data show that MDM2 gene expression in A549 cells is specifically suppressed by MDM2 siRNA1 and MDM2 siRNA2 with the greater level of suppression by siRNA1. MDM2 siRNA1-transfected cells combined with 5-Gy IR significantly inhibited cell proliferation. Also, siRNA1 significantly increased IR-mediated cell death by increasing apoptosis as well as necrosis $(45.9 \%$ and $15.2 \%$ compared to $2.6 \%$ and $14.4 \%$, respectively, for IR alone). Therefore, a combination of MDM2 siRNA1 with radiotherapy would be a preferential strategy to enhance the therapeutic efficacy for radioresistant lung cancers.

\section{Materials and methods}

Construction of MDM2 siRNA plasmids. The sequences of the following two different MDM2-specific siRNAs were determined from the human MDM2 mRNA sequence (GenBank Accession no. XM_017531). MDM2 siRNA1: 5'-GTGCTGG GATTACAGGCATTTCAAGAGAATGCCTGTAATCCC AGCACTTTTTT-3' (sense); 5'-AATTAAAAAAGTGCTGG GATTACAGGCATTCTCTTGAAATGCCTGTAATCCCA GCACGGCC-3' (antisense). MDM2 siRNA2: 5'-TCACCTT GAAGGTGGGAGTTTCAAGAGAACTCCCACCTTCAA GGTGATTTTTT-3' (sense); 5'-AATTAAAAAATCACCTT GAAGGTGGGAGTTCTCTTGAAACTCCCACCTTCAAG GTGAGGCC-3' (antisense). To construct MDM2 siRNA plasmids, equal amounts of the sense and antisense templates were annealed, and the duplex oligonucleotides were inserted into the ApaI and EcoRI sites of pUR/U6 vector (a kind gift from Dr Z.Y. Shen, Cancer Institute of New Jersey UMDNJ Robert Wood Johnson Medical School, USA). All constructs were sequence-verified before use.

Cell culture and transient transfection. A549 lung cancer cells were maintained as adherent monolayer cultures in RPMI-1640 culture medium (HyClone, Logan, UT), supplemented with $10 \%$ fetal bovine serum, $100 \mathrm{U} / \mathrm{ml}$ penicillin and $100 \mu \mathrm{g} / \mathrm{ml}$ streptomycin in a humidified chamber at $37^{\circ} \mathrm{C}$ in $5 \% \mathrm{CO}_{2}$. Cells grown in 6-well plates were transfected with $3 \mu \mathrm{g}$ of MDM2 siRNA plasmids or pUR/U6 empty plasmid as a vector control, using Lipofectamine ${ }^{\mathrm{TM}} 2000$ (Invitrogen, Long Island, NY, USA) according to the manufacturer's protocol. Cells were fed fresh medium $72 \mathrm{~h}$ post-transfection.

Irradiation. Cells grown in 6-well plates were irradiated with a single dose of 5 Gy under ambient conditions. All irradiations were performed at a ${ }^{60} \mathrm{Co}$ unit with a dose rate of 1.51-1.68 Gy/min ${ }^{-1}$. Plates containing control cells were taken to the radiation chamber but not those exposed to IR serving as the sham-IR control. The culture media of irradiated and sham-irradiated cells were replaced with fresh medium immediately after IR.

$R T-P C R$. Total RNA was extracted from A549 cells using TRIzol reagent (Invitrogen) at $72 \mathrm{~h}$ post-transfection with MDM2 siRNA plasmids. The concentration and purity of the RNA samples were determined spectroscopically. cDNA was synthesized according to the protocol of ImProm-II ${ }^{\mathrm{TM}}$ reverse transcription system (Promega, Madison, WI, USA). The newly synthesized cDNA was amplified by PCR. The primer sequences for MDM2 were forward, 5'-AAG ACT ATT CTC AGC CAT CA-3'; reverse, 5'-CAT ACT GGG CAG GGC ATT-3'. As internal standard, human endogenous GAPDH was amplified simultaneously. The primer sequences for GAPDH were forward, 5'-ATC CCA TCA CCA TCT TCC AG-3'; reverse, 5'-CCA TCA CGC CAC AGT TTC C-3'. The predicted size of the PCR products for MDM2 and GAPDH are 259 and $363 \mathrm{bp}$, respectively. Amplification cycles were: $94^{\circ} \mathrm{C}$ for $5 \mathrm{~min}$, then 30 cycles at $94^{\circ} \mathrm{C}$ for $30 \mathrm{sec}$, $55^{\circ} \mathrm{C}$ for $30 \mathrm{sec}, 72^{\circ} \mathrm{C}$ for $40 \mathrm{sec}$, followed by a final elongation at $72^{\circ} \mathrm{C}$ for $5 \mathrm{~min}$. Aliquots of PCR products were electrophoresed on a $2 \%$ agarose gel. PCR fragments were visualized by ethidium bromide staining and quantified with an image analyzer (Quantity One; Bio-Rad, Hercules, CA, USA).

Western blot analysis. Protein levels of MDM2 as well as normalization control $\mathrm{B}$-actin were determined with or without IR treatment. A549 cells were transfected with expression vector for MDM2 siRNA1 or empty vector (pUR/U6) for $72 \mathrm{~h}$. The transfected cells were exposed to $5 \mathrm{~Gy}$ of $\gamma$-irradiation using a ${ }^{60} \mathrm{Co}$ irradiator and incubated for $48 \mathrm{~h}$. Cells were then washed twice in ice-cold PBS, and lysed in mammalian cell lysis buffer (10 mM sodium phosphate $\mathrm{pH} 7.5,100 \mathrm{mM}$ $\mathrm{NaCl}, 1 \%$ Triton $\mathrm{X}-100,0.5 \%$ deoxycholate, $1 \mathrm{mM}$ phenylmethylsulfonyfluoride, and $15 \mathrm{mg} / \mathrm{ml}$ aprotinin). The lysates were transferred to new tubes and clarified by centrifugation at $5000 \mathrm{x} \mathrm{g}$ for $10 \mathrm{~min}$ at $4^{\circ} \mathrm{C}$. Protein concentration was determined using the BCA protein assay reagent kit (Pierce, Rockford, IL, USA). Identical amounts of protein were resolved by SDS-PAGE and blotted onto the polyvinylidene difluoride (PVDF) membranes (Millipore, Bedford, MA, USA). After electroblotting, the membranes were incubated in blocking buffer (phosphate-buffered saline containing 0.1\% Tween-20 and $5 \%$ non-fat milk) for $1 \mathrm{~h}$ at room temperature, then immunoblotted with anti-MDM2 monoclonal antibody (1:500, Santa Cruz Biotechnology, Santa Cruz, CA, USA) overnight at $4^{\circ} \mathrm{C}$. The membranes were washed, incubated with the sheep anti-mouse IgG horseradish peroxidaseconjugated secondary antibody (1:2500, Santa Cruz Biotechnology) for $1 \mathrm{~h}$ at room temperature, and washed again. The blotted proteins were detected by the Western Blotting Luminol Reagent (Santa Cruz Biotechnology) according to the manufacturer's directions. The membrane probed for MDM2 was stripped and reprobed for B-actin $(1: 1000$, Santa Cruz Biotechnology) to normalize for loading and/or quantification errors.

MTT assay. The effects on cell growth by the MDM2 siRNA1 were examined by MTT assay. Briefly, cells were seeded at a density of 5000 cells/well in 96-well plates and allowed to attach overnight. Cells were then transfected with pUR/U6 vector control or pUR/U6 containing MDM2 siRNA1, and irradiated $72 \mathrm{~h}$ post-transfection. After $48 \mathrm{~h}$ of irradiation, $20 \mu 1$ of MTS solution (Sigma, St. Louis, MO, USA) was added to each well, and the cells were incubated for $90 \mathrm{~min}$ at $37^{\circ} \mathrm{C}$. The absorbance of each well was measured using a MR 600 Microplate reader (Wallac 1420 Victor 2 multilable 

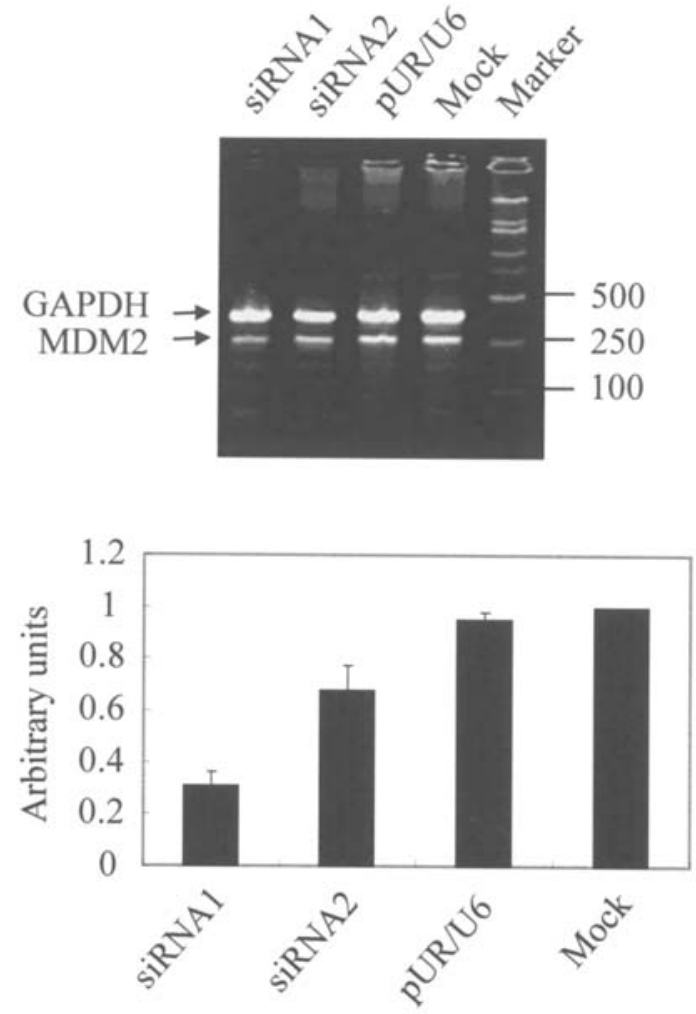

Figure 1. Effect of siRNAs on MDM2 mRNA in A549 cells. For the selection of an effective siRNA directed against MDM2, A549 cells were transfected with the mock (lipofectamine), pUR/U6 empty vector or MDM2-specific siRNA vectors. Seventy-two hours after transfection, the total RNA was extracted and mRNA expression was detected by semi-quantitative RTPCR, as described in Materials and methods. Each PCR was run in triplicate. GAPDH mRNA was also amplified as an internal control. Lower panel shows the relative MDM2 mRNA expression levels estimated by densitometry and normalized to control GAPDH.

counter system, Gaithersburg, MD, USA) at $490 \mathrm{~nm}$. All samples were made in triplicate, and the data were analyzed using the Student's t-test.

Flow cytometry analysis. A549 cells were transfected with pUR/U6 expression vector for MDM2 siRNA1 or empty vector for $72 \mathrm{~h}$ before irradiation ( 5 Gy). Cells were harvested $48 \mathrm{~h}$ post-radiation, washed twice in PBS, and stained with Annexin V-FITC/PI (Baosai Reagent, P.R. China). The apoptotic and necrotic cells were assessed by flow cytometric detection using a FACSCalibur and analyzed with CellQuest software program (Becton Dickinson, San Jose, CA, USA).

\section{Results}

siRNA-mediated down-regulation of MDM2. We designed siRNA complementary to two different regions of the MDM2 mRNA as described in Materials and methods. Each siRNA was synthesized as complementary oligonucleotides and cloned into the pUR/U6 expression vector. The resulting constructs were then screened for their ability to down-regulate MDM2 expression. A549 cells were transfected with empty pUR/U6 vector or the vector for the expression of each siRNA, and RT-PCR was performed $72 \mathrm{~h}$ post-transfection. As shown in Fig. 1, siRNA1 and siRNA2 directed against MDM2 reduced
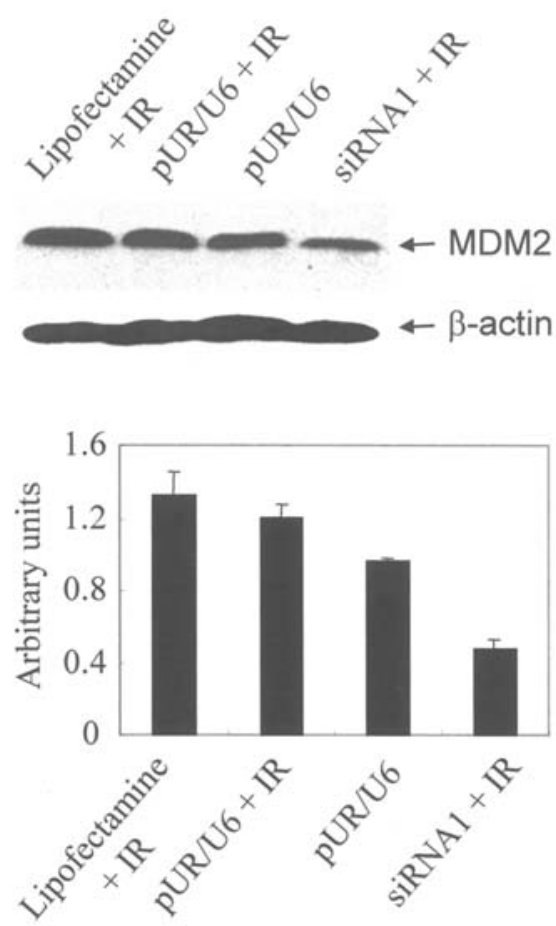

Figure 2. Effect of siRNA1 on MDM2 protein expression in A549 cells treated with IR. Cells were transfected with the mock, pUR/U6 or MDM2specific siRNA1 vector before exposure to 5-Gy IR. Forty-eight hours post-radiation, the cell lysates were prepared and Western blot analysis was performed with anti-MDM 2 antibody, and reprobed with $ß$-actin antibody as a control. Lower panel shows the relative MDM2 protein levels estimated using densitometry and normalized to control B-actin.

MDM2 mRNA levels by $72 \%$ and $31 \%$, respectively, compared to GAPDH, which was not affected by either siRNA. Although both siRNAs attenuated MDM2 expression significantly, the greatest level of suppression was observed by siRNA1. Therefore, MDM2 siRNA1 was used for further studies.

Next, Western blot analysis was performed to determine the protein levels of MDM2 in A549 cells transfected with expression vector for MDM2 siRNA1 followed by exposure to $\gamma$-irradiation (5 Gy). We found that, compared to $\beta$-actin, IR-mediated induction of MDM2 expression (Fig. 2, lanes 1 and 2) was greatly reduced (69\%) by siRNA1 directed against MDM2 (Fig. 2, lane 4). The reduction in protein levels is very similar to the reduction of mRNA levels shown in Fig. 1 (69\% versus $72 \%$ ). Also, compared to vector (pUR/U6)transfected control cells (Fig. 2, lane 3), MDM2 level was much lower (52\%) in A549 cells transfected with siRNA1 before exposure to 5-Gy IR (Fig. 2, lane 4). Together with Fig. 1, Fig. 2 clearly demonstrates that MDM2 siRNA1 was highly specific and efficient in MDM2 gene silencing at both the mRNA and protein levels in A549 cells.

siRNA-mediated MDM2 inhibition sensitizes A549 cells to $I R$. To determine if inhibition of MDM2 radiosensitizes A549 cells, cell proliferation was measured with MTT assay. Cells were transfected for $72 \mathrm{~h}$ with expression vector for MDM2 siRNA1 or vector alone before exposure to 5-Gy IR. Two days post-radiation, MTT assay was performed. As shown in Fig. 3, siRNA1-transfected A549 cells with 5-Gy 


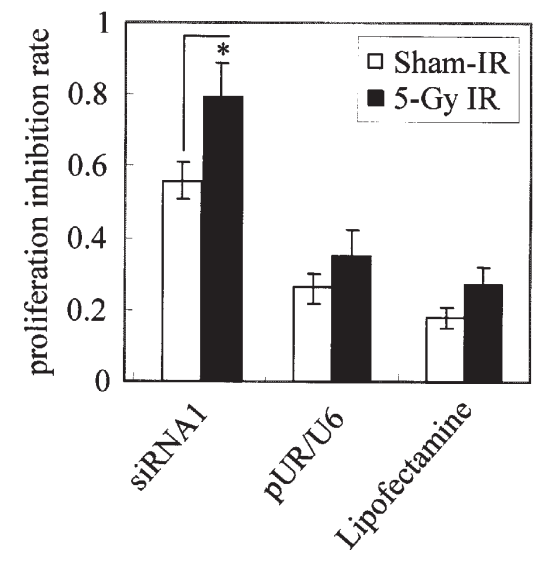

Figure 3. Effects of siRNA1-mediated silencing of MDM2 on the radiosensitivity of A549 cells. Cells were transfected with the mock, pUR/U6 or MDM2-specific siRNA1 vector before exposure to or without 5-Gy IR. The cell proliferation was determined $48 \mathrm{~h}$ post-radiation by MTT colorimetric assay. Each bar represents the relative cell growth inhibition (mean \pm standard deviations) from three independent experiments. ${ }^{*} \mathrm{p}<0.01$, compared to A549 cells transfected with vector for siRNA1 expression before shamirradiation.

Table I. Flow cytometry analysis of the effects of MDM2 siRNA1 on cell death of A549 cells with or without 5-Gy IR.

\begin{tabular}{lcr}
\hline Group & Apoptosis & Necrosis \\
\hline A549 & $0.8 \pm 0.2$ & $2.1 \pm 0.6$ \\
A549 + IR & $2.6 \pm 0.6$ & $14.4 \pm 3.5$ \\
A549 + pUR/U6 & $3.62 \pm 1.1$ & $4.8 \pm 0.8$ \\
A549 + siRNA1 & $30.1 \pm 7.5^{\mathrm{a}}$ & $12.7 \pm 4.8^{\mathrm{a}}$ \\
A549 + siRNA1 + IR & $45.9 \pm 8.2^{\mathrm{a}}$ & $15.2 \pm 6.3^{\mathrm{a}}$ \\
\hline
\end{tabular}

${ }^{\mathrm{a}} \mathrm{p}<0.01$, compared to A549 + pUR/U6, by One-way ANOVA test for apoptosis and necrosis.

IR showed reduced proliferation compared to cells with exposure to sham-IR $(\mathrm{p}<0.01)$. The IR-induced inhibition of cell proliferation was not significant although cells appeared to be more inhibited when IR was combined with transfection of vector (pUR/U6) or mock (lipofectamine). These data suggest that siRNA1-mediated silencing of MDM2 significantly inhibits cell proliferation and renders A549 cells more sensitive to radiation.

To determine whether depletion of MDM2 by siRNA1 could promote the death of tumor cells, flow cytometry was performed. At $72 \mathrm{~h}$ after transfection with MDM2 siRNA1 expression vector, A549 cells were exposed to 5-Gy IR. Forty-eight hours post-radiation, cells were labeled with Annexin V-FITC/PI and analyzed with a FACSCalibur flow cytometer. The basal apoptotic and necrotic cells, $1 \%$ and $2 \%$, respectively, detected in wild-type A549 cells were increased to $2.6 \%$ and $14.4 \%$ by 5 -Gy $\gamma$-ray (Table I). MDM2 siRNA1 increased the number of apoptotic as well as necrotic cells by $30.1 \%$ and $12.7 \%$, respectively, compared to $3.6 \%$ and $4.8 \%$, respectively, observed in empty pUR/U6 vector-transfected
A549 cells $(\mathrm{p}<0.01)$. Interestingly, IR further increased the number of both apoptotic and necrotic cells by $45.9 \%$ and $15.2 \%$, respectively, in MDM2 siRNA1 transfected cells $(\mathrm{p}<0.01)$. These results suggest that the sensitivity of MDM2 siRNA1 expressing A549 cells to DNA damage caused by 5-Gy IR was significantly increased.

\section{Discussion}

Clinically, many non-small cell lung carcinomas (NSCLCs) are inoperable, and little tumor-controlling efficacy is achieved by radiotherapy or chemotherapy, or a combination of both therapies due to tumor resistance. Therefore, developing new therapies to improve the overall outcome of NSCLC patients is in urgent need (20). Although potential targets for therapeutic intervention in therapy-resistant lung cancers have been extensively studied in the last decade $(21,22)$, no efficient molecular target has been identified.

Our present results demonstrate for the first time that inhibition of MDM2 with a specific human siRNA sequence (MDM2 siRNA1) is able to re-sensitize radiation-resistant lung cancer A549 cells. Oncogenes provide a potential target for cancer gene therapy (23). Overexpression of the oncogene MDM2 is a common event associated with the pathogenesis of most human cancers $(24,25)$, including breast cancer, osteosarcomas and lung cancer $(13,14,26-28)$. Activation of MDM2 increases the expression of genes that promote cell survival and block apoptosis (25). Transduction of MDM2-b (an alternative splice form of MDM2 most commonly observed in human cancers) into NIH3T3 cells can promote rapid cell proliferation (29). Interestingly, tumor regression was observed by the inactivation of MDM2 (30). Overexpression of MDM2 in various tumors is believed to inhibit $\mathrm{p} 53$, therefore favoring uncontrolled cell proliferation. We reported that MDM2 expression levels were increased in non-small cell lung carcinoma A549 cells in response to IR (9). Therefore, specific down-regulation of MDM2 may be a potential target to enhance radiosensitization.

Other groups have applied antisense to attenuate MDM2 expression resulting in the inhibition of proliferation of cancer cells in vitro (31-33). Our previous study also demonstrated that MDM2 ASODN (antisense oligodeoxynucleotide) enhanced radiosensitivity in A549 cells (9). Although antisense oligonucleotides are efficient to block MDM2, RNA interference is a powerful technique for the specific inhibition of expression of individual genes at the posttranscriptional level (34). siRNA has a number of advantages over antisense oligonucleotide strategy, in part from the greater resistance of siRNA to nuclease degradation. RNAi and antisense RNA have been compared and RNAi seemed to be quantitatively more efficient and durable in both cell culture and nude mice (35). Therefore, the feasibility of using siRNA in molecular cancer therapy and as a mediator of chemosensitization or radiosensitization has been shown by several groups $(36,37)$.

In the present study, the functional consequences of siRNA-mediated suppression of MDM2, using expression pUR/U6 vectors for MDM2 siRNA1 and MDM2 siRNA2, was established in radioresistant A549 non-small cell lung cancer cells. Our results demonstrated that inhibition of MDM2 by siRNA1 enhanced response to radiation, which was 
associated with significant reduced levels of cell proliferation (Fig. 3) as well as increased levels of apoptosis (45.9\% and $2.6 \%$ for dual treatment of siRNA1 and IR, and IR alone, respectively; Table I). As a result, there was enhanced radiosensitivity in A549 cells. Although synthetic siRNAs can achieve effective and very rapid knockdown of target genes in mammalian cells, their effects are transient. To circumvent this problem, transfection of siRNA plasmid that stably integrates and expresses interfering double-strand RNA hairpins may be effective at knocking down target genes continually. In this study, although MDM2 siRNA was transfected transiently in puromycin-resistant A549 cells, we surprisingly found loss of long-term gene silencing (data not shown). Because of the loss of long-term gene silencing, the clonogenicity assay was not performed. However, in our previous studies, a correlation between clonogenic radiosensitivity and apoptotic cells was found $(38,39)$.

In conclusion, we have tested two MDM2 siRNAs (i.e., siRNA1 and siRNA2), and found different inhibition efficiency as determined by both mRNA and protein levels (Figs. 1, 2 and data not shown). Accumulating evidence suggest that different regions of mRNA show a varied efficacy of gene inhibition by siRNA-mediated targeting. Therefore, it appears that the region of siRNA2 is not an efficient target for MDM2. While the manuscript of this article was under preparation, Yan Yu et al described increased resistance of human colorectal adenocarcinoma LoVo cells to the DNA damaging agent cisplatin after treatment with MDM2 siRNA (40), a result supportive to our conclusion. The most efficient MDM2 siRNA sequence (i.e., siRNA3) demonstrated by Yu et al (40) is different from the potential target sequence (i.e., siRNA1) observed in the current study, although they found $80 \%$ reduction of tumor growth in combination with cisplatin. Therefore, double siRNA treatment (i.e., MDM2 siRNA1 of this study and MDM2 siRNA3 of ref. 40) in combination with radiotherapy may provide a more efficient strategy in tumor radiosensitization. Overall, the data presented here provide evidence suggesting that MDM2 siRNA could be a promising and powerful method for sensitization of radioresistant cancer cells.

\section{References}

1. Spira A and Ettinger DS: Multidisciplinary management of lung cancer. N Engl J Med 350: 379-392, 2004.

2. Komaki R, Liao $\mathrm{Z}$ and Milas L: Improvement strategies for molecular targeting: cyclooxygenase-2 inhibitors as radiosensitizers for non-small cell lung cancer. Semin Oncol 31: 47-53, 2004.

3. Dy GK and Adjei AA: Novel targets for lung cancer therapy: part I. J Clin Oncol 20: 2881-2894, 2002.

4. Stassen T, Port M, Nuyken I and Abend M: Radiation-induced gene expression in MCF-7 cells. Int J Radiat Biol 79: 319-331, 2003.

5. Yin E, Nelson DO, Coleman MA, Peterson LE and Wyrobek AJ: Gene expression changes in mouse brain after exposure to low-dose ionizing radiation. Int J Radiat Biol 79: 759-775, 2003.

6. Lehnert S: Prediction of tumor response to therapy: molecular markers and the microenvironment. Apoptosis and chips: an overview of the proceedings. Radiat Res 154: 121-124, 2000.

7. Guo G, Yan-Sanders Y, Lyn-Cook BD, Wang T, Tamae D, Ogi J, Khaletskiy A, Li Z, Weydert C, Longmate JA, Huang TT, Spitz DR, Oberley LW and Li JJ: Manganese superoxide dismutase-mediated gene expression in radiation-induced adaptive responses. Mol Cell Biol 23: 2362-2378, 2003.
8. Xia L, Paik A and Li JJ: p53 activation in chronic radiationtreated breast cancer cells: regulation of MDM2/p14ARF. Cancer Res 64: 221-228, 2004.

9. Guo WF, Lin RX, Huang J, Zhou Z, Yang J, Guo GZ and Wang SQ: Identification of differentially expressed genes contributing to radioresistance in lung cancer cells using microarray analysis. Radiat Res 164: 27-35, 2005.

10. Perry ME: Mdm2 in the response to radiation. Mol Cancer Res 2: 9-19, 2004.

11. Iwakuma T and Lozano G: MDM2, an introduction. Mol Cancer Res 1: 993-1000, 2003.

12. Ganguli G and Wasylyk B: p53-independent functions of MDM2. Mol Cancer Res 1: 1027-1035, 2003.

13. Oliner JD, Kinzler KW, Meltzer PS, George DL and Vogelstein B: Amplification of a gene encoding a p53-associated protein in human sarcomas. Nature 358: 80-83, 1992.

14. Cordon-Cardo C, Latres E, Drobnjak M, Oliva MR, Pollack D, Woodruff JM, Marechal V, Chen J, Brennan MF and Levine AJ: Molecular abnormalities of mdm2 and p53 genes in adult soft tissue sarcomas. Cancer Res 54: 794-799, 1994.

15. Gorgoulis VG, Zacharatos P, Kotsinas A, Liloglou T, Kyroudi A, Veslemes M, Rassidakis A, Halazonetis TD, Field JK and Kittas C: Alterations of the p16-pRb pathway and the chromosome locus 9p21-22 in non-small-cell lung carcinomas: relationship with p53 and MDM2 protein expression. Am J Pathol 153: 1749-1765, 1998

16. Valassiadou KE, Stefanaki K, Tzardi M, Datseris G, Georgoulias V, Melissas J, Tsiftsis DD, Delides G and Kanavaros P: Immunohistochemical expression of p53, bcl-2, $\mathrm{mdm} 2$ and waf $1 / \mathrm{p} 21$ proteins in colorectal adenocarcinomas. Anticancer Res 17: 2571-2576, 1997.

17. Sjostrom J, Blomqvist C, Heikkila P, Boguslawski KV, Raisanen-Sokolowski A, Bengtsson NO, Mjaaland I, Malmstrom P, Ostenstadt B, Bergh J, Wist E, Valvere V and Saksela E: Predictive value of p53, mdm-2, p21, and mib-1 for chemotherapy response in advanced breast cancer. Clin Cancer Res 6: 3103-3110, 2000.

18. Elbashir SM, Harborth J, Lendeckel W, Yalcin A, Weber K and Tuschl T: Duplexes of 21-nucleotide RNAs mediate RNA interference in cultured mammalian cells. Nature 411: 494-498, 2001.

19. Lewis DL, Hagstrom JE, Loomis AG, Wolff JA and Herweijer H: Efficient delivery of siRNA for inhibition of gene expression in postnatal mice. Nat Genet 32: 107-108, 2002.

20. Lammering G, Hewit TH, Valerie K, Lin PS, Contessa JN and Schmidt-Ullrich RK: Anti-erbB receptor strategy as a gene therapeutic intervention to improve radiotherapy in malignant human tumours. Int J Radiat Biol 79: 561-568, 2003.

21. Rosenberg SA: Gene therapy for cancer. JAMA 268: 2416-2419, 1992.

22. Hoang T, Traynor AM and Schiller JH: Novel therapies for lung cancer. Surg Oncol 11: 229-241, 2002.

23. Buolamwini JK, Addo J, Kamath S, Patil S, Mason D and Ores M: Small molecule antagonists of the MDM2 oncoprotein as anticancer agents. Curr Cancer Drug Targets 5: 57-68, 2005.

24. Onel K and Cordon-Cardo C: MDM2 and prognosis. Mol Cancer Res 2: 1-8, 2004.

25. Slack A and Shohet JM: MDM2 as a critical effector of the MYCN oncogene in tumorigenesis. Cell Cycle 4: 857-860, 2005.

26. McCann AH, Kirley A, Carney DN, Corbally N, Magee HM, Keating $\mathrm{G}$ and Dervan PA: Amplification of the MDM2 gene in human breast cancer and its association with MDM2 and p53 protein status. Br J Cancer 71: 981-985, 1995.

27. Marchetti A, Buttitta F, Girlando S, Dalla Palma P, Pellegrini S, Fina P, Doglioni C, Bevilacqua $\mathrm{G}$ and Barbareschi M: $\mathrm{mdm} 2$ gene alterations and $\mathrm{mdm} 2$ protein expression in breast carcinomas. $\mathrm{J}$ Pathol 175: 31-38, 1995.

28. Dworakowska D, Jassem E, Jassem J, Peters B, Dziadziuszko R, Zylicz M, Jakobkiewicz-Banecka J, Kobierska-Gulida G, Szymanowska A, Skokowski J, Roessner A and SchneiderStock R: MDM2 gene amplification: a new independent factor of adverse prognosis in non-small cell lung cancer (NSCLC). Lung Cancer 43: 285-295, 2004.

29. Steinman HA, Burstein E, Lengner C, Gosselin J, Pihan G, Duckett CS and Jones SN: An alternative splice form of Mdm2 induces p53-independent cell growth and tumorigenesis. J Biol Chem 279: 4877-4886, 2004

30. Momand J, Jung D, Wilczynski S and Niland J: The MDM2 gene amplification database. Nucleic Acids Res 26: 3453-3459, 1998. 
31. Chene P: Inhibiting the p53-MDM2 interaction: an important target for cancer therapy. Nat Rev Cancer 3: 102-109, 2003.

32. Mu Z, Hachem P, Agrawal S and Pollack A: Antisense MDM2 sensitizes prostate cancer cells to androgen deprivation, radiation, and the combination. Int J Radiat Oncol Biol Phys 58: 336-343, 2004.

33. Sato N, Mizumoto K, Maehara N, Kusumoto M, Nishio S, Urashima T, Ogawa T and Tanaka M: Enhancement of druginduced apoptosis by antisense oligodeoxynucleotides targeted against Mdm2 and p21WAF1/CIP1. Anticancer Res 20: 837-842, 2000.

34. Shi Y: Mammalian RNAi for the masses. Trends Genet 19: 9-12, 2003.

35. Tan FL and Yin JQ: RNAi, a new therapeutic strategy against viral infection. Cell Res 14: 460-466, 2004.

36. Collis SJ, Swartz MJ, Nelson WG and De Weese TL: Enhanced radiation and chemotherapy-mediated cell killing of human cancer cells by small inhibitory RNA silencing of DNA repair factors. Cancer Res 63: 1550-1554, 2003.
37. Wu H, Hait WN and Yang JM: Small interfering RNA-induced suppression of MDR1 (P-glycoprotein) restores sensitivity to multidrug-resistant cancer cells. Cancer Res 63: 1515-1519, 2003.

38. Guo GZ, Sasai K, Oya N, Takagi T, Shibuya K and Hiraoka M: Simultaneous evaluation of radiation-induced apoptosis and micronuclei in five cell lines. Int J Radiat Biol 73: 297-302, 1998.

39. Guo GZ, Sasai K, Oya N, Shibata T, Shibuya K and Hiraoka MA Significant correlation between clonogenic radiosensitivity and the simultaneous assessment of micronucleus and apoptotic cell frequencies. Int J Radiat Biol 75: 857-864, 1999.

40. Yu Y, Sun P, Sun LC, Liu GY, Chen GH, Shang LH, Wu HB, $\mathrm{Hu}$ J, Li Y, Mao YL, Sui GJ and Sun XW: Downregulation of MDM2 expression by RNAi inhibits LoVo human colorectal adenocarcinoma cells growth and the treatment of LoVo cells with mdm2siRNA3 enhances the sensitivity to cisplatin. Biochem Biophys Res Commun 339: 71-78, 2006. 